\title{
Polygonatum sibiricum polysaccharide alleviates inflammatory cytokines and promotes glucose uptake in high-glucose- and high-insulin-induced 3T3-L1 adipocytes by promoting Nrf2 expression
}

\author{
JIALUO CAI $^{1^{*}}$, YILIN ZHU ${ }^{2 *}$, YAJIE ZUO ${ }^{3}$, QIAOZHEN TONG ${ }^{4,5}$, ZHIGUO ZHANG $^{3}$, \\ LEI YANG ${ }^{6}$, XIAOPING LI ${ }^{1}$ and GANGQIANG YI $^{5}$ \\ ${ }^{1}$ Preventive Treatment of Disease Center, The First Hospital of Hunan University of Chinese Medicine; \\ ${ }^{2}$ Student Affairs Office, Hunan University of Chinese Medicine; ${ }^{3}$ Department of Pharmacy, \\ The First Hospital of Hunan University of Chinese Medicine, Changsha, Hunan 410000; \\ ${ }^{4}$ Yueyang Affiliated Hospital of Hunan University of Chinese Medicine, Yueyang, Hunan 414000; \\ ${ }^{5}$ Hunan University of Chinese Medicine; ${ }^{6}$ Preparation Center, The First Hospital of Hunan \\ University of Chinese Medicine, Changsha, Hunan 410000, P.R. China
}

Received April 17, 2019; Accepted July 31, 2019

DOI: $10.3892 / \mathrm{mmr} .2019 .10626$

\begin{abstract}
Polygonatum sibiricum polysaccharide (PSP) has been shown to alleviate hyperglycemia and reduce oxidative stress to delay the progression of diabetic retinopathy and cataracts. However, its role and underlying mechanisms in regulating type 2 diabetes mellitus (T2DM) remain unclear. Nuclear factor erythroid 2-related factor 2 (Nrf2) activation plays a protective role in T2DM. The present study focused on the effect of PSP on inflammatory cytokine secretion and Nrf2 expression in the adipocytes of T2DM patients. In this study, high-glucose- and high-insulin-induced 3T3-L1 adipocytes were used to mimic insulin-resistant (IR)-3T3-L1 adipocytes. Furthermore, the effect and underlying mechanisms of PSP on inflammation and glucose uptake in IR-3T3-L1 adipocytes were investigated. The present study found that proliferation after 50, 100 and $250 \mu \mathrm{g} / \mathrm{ml}$ PSP treatment had no significant change in normal 3T3-L1 adipocytes. A total of 50, 100 and
\end{abstract}

Correspondence to: Ms. Xiaoping Li, Preventive Treatment of Disease Center, The First Hospital of Hunan University of Chinese Medicine, 95 Shaoshan Middle Road, Changsha, Hunan 410000, P.R. China

E-mail: 1xp1074772037@sina.com

Dr Gangqiang Yi, Hunan University of Chinese Medicine, 300 Bachelor's Road, Changsha, Hunan 410000, P.R. China

E-mail:ygq8228@163.com

*Contributed equally

Key words: Polygonatum sibiricum polysaccharide, insulin resistance, type 2 diabetes mellitus, adipocytes, nuclear factor erythroid 2-related factor 2
$250 \mu \mathrm{g} / \mathrm{ml}$ of PSP also alleviated IL-1 $\beta$, IL-6, and TNF- $\alpha$ levels and promoted proliferation, glucose uptake, and glucose transporter 4 expression in IR-3T3-L1 adipocytes. Furthermore, 50, 100 and $250 \mu \mathrm{g} / \mathrm{ml}$ PSP promoted Nrf2 and HO-1 expression. However, silencing Nrf2 expression reversed the effect of $100 \mu \mathrm{g} / \mathrm{ml}$ PSP in IR-3T3-L1 adipocytes. In conclusion, these results suggest that PSP alleviates inflammatory cytokines and promotes glucose uptake in IR-3T3-L1 adipocytes by promoting Nrf2 expression. PSP may be a potential therapeutic agent for T2DM treatment by promoting Nrf2 expression.

\section{Introduction}

Type 2 diabetes mellitus (T2DM), a serious public health issue worldwide, is harmful to important organs such as the eyes, kidneys and nerves due to insulin resistance (IR) and high glucose concentrations in the blood (1). Currently, T2DM treatment has advanced considerably; however, the available treatments remain limited. Numerous studies have strongly associated obesity with T2DM $(2,3)$. Notably, dysfunctional adipocytes, regulated the excessive secretion of inflammatory cytokines, represent a key link between obesity and IR $(4,5)$. Moreover, clinical and epidemiological studies also associated the development of T2DM with inflammatory factors, which are new targets for T2DM treatment (6). Therefore, it will be important to identify new drugs that can inhibit proinflammatory adipocytokine secretion in T2DM.

Traditional Chinese medicines (TCMs) have exhibited great efficacy in treating T2DM with good application prospects. Chen et al (6) found that berberine markedly prevented the development of T2DM by modulating Bifidobacterium species and inhibiting inflammation and development of diabetic nephropathy. Furthermore, puerarin was found to reduce inflammation and blood glucose levels and improve IR, thereby improving the development of T2DM and DM 
complications (7). The Huang-Lian-Jie-Du decoction, a TCM prescription, also regulated gut microbiota and improved hyperglycemia and IR in T2DM (8). Polygonatum sibiricum polysaccharide (PSP) is isolated from Polygonatum sibiricum. Additionally, water-soluble extractions of PSP are of low toxicity and are suitable for long-term administration (9). The hot water-extraction of PSP product is composed of different ratios of monosaccharides, including rhamnose, mannose, xylose, arabinose, fucose, galactose, glucose, galacturonic acid and glucuronic acid $(10,11)$. Polygonatum sibiricum and PSP have many pharmacological applications and biological activities and has used to treat a number of diseases $(9,10)$. It is reported that PSP could inhibit inflammation such as interleukin (IL)-1 $\beta$, IL-6 and tumor necrosis factor (TNF)- $\alpha$ expression in lipopolysaccharide-treatment RAW264.7, ovariectomized rat, acute heart failure rats, suggest that PSP had anti-inflammation effect (12-14). Moreover, PSP alleviated hyperglycemia and reduced oxidative stress to delay the progression of diabetic retinopathy and cataracts $(15,16)$. Therefore, more studies are needed to further confirm the role and mechanism of PSP in regulating diabetes, especially T2DM. However, the regulating effect of PSP on inflammation and glucose uptake in regulating T2DM remains unclear.

Nrf2 plays a key role in regulating inflammation (17). Nrf2 and Kelch-like ECH-associated protein 1 are uncoupled to allow Nrf2 localization in the nucleus, then Nrf2 binds to the heme oxygenase 1 (HO-1) promoter to promote $\mathrm{HO}-1$ expression, which can activate nuclear factor- $\mathrm{\kappa B}$ and promote pro-inflammatory secretion (18-20). Nrf2 could regulate metabolic homeostasis and insulin action and it may serve as a potential drug target for diabetes and treatment of its complications (21). However, whether PSP can improve the IR of 3T3-L1 adipocytes through Nrf2 is still unclear.

In this study, the effect of PSP on inflammatory cytokine secretion and glucose uptake in high-glucose- and high-insulin-induced 3T3-L1 adipocytes to mimic IR-3T3-L1 adipocytes was focused on. Furthermore, the underlying mechanisms of PSP on inflammation and glucose uptake in IR-3T3-L1 adipocytes was investigated. In present study, whether PSP alleviated inflammatory cytokines and promotes glucose uptake in high-glucose- and high-insulin-induced 3T3-L1 adipocytes by promoting Nrf2 expression was investigated.

\section{Materials and methods}

The compete experimental schedule is shown in Fig. 1.

Drugs. PSP was purchased from Shaanxi Undersun Biomedtech Co., Ltd. PSP was pretreated and extracted using a hot water extraction method. Its purity was $92 \%$ according to the certificate of analysis. PSP was dissolved in $1 \mathrm{mg} / \mathrm{ml}$ of $0.1 \mathrm{~mol} / \mathrm{l} \mathrm{PBS}$ prior to use.

Cell culture and establishment of an IR model. 3T3-L1 pre-adipocytes (American Type Cell Culture) were cultured and induced to differentiate into mature adipocytes as described previously (22). To establish an IR model (IR-3T3-L1 adipocytes), 3T3-L1 adipocytes were cultured for $24 \mathrm{~h}$ at $37^{\circ} \mathrm{C}$ in Dulbecco's modified Eagle's medium (Gibco; Thermo Fisher
Scientific, Inc.) supplemented with $10 \%$ fetal bovine serum (Gibco; Thermo Fisher Scientific, Inc.), $1 \mu \mathrm{mol} / 1$ insulin and $25 \mathrm{mmol} / \mathrm{l}$ glucose.

Proliferation assay. Proliferation of IR-3T3-L1 adipocytes was measured using Cell Counting Kit-8 (CCK-8; Beyotime Institute of Biotechnology) assay. In total, $1 \times 10^{4}$ IR-3T3-L1 adipocytes/well were cultured and treated with $0,50,100$, 250,500 and $1,000 \mu \mathrm{g} / \mathrm{ml}$ of PSP for 12, 24 and $48 \mathrm{~h}$. CCK- 8 $(10 \mu \mathrm{l})$ was then added and incubated for $4 \mathrm{~h}$. Absorbance was detected at $450 \mathrm{~nm}$ using a microplate absorbance reader (R\&D Systems, Inc.). Each experiment was repeated thrice.

Inflammatory cytokine and glucose assay. IL-1 1 , IL-6 and TNF- $\alpha$ levels in culture supernatants were measured using ELISA according to the protocol supplied by the manufacturer [cat. nos. EK101B2-96T, EK1062-96T and EK1822-96T; Hangzhou MultiSciences (Lianke) Biotech, Co., Ltd.]. Glucose levels in culture supernatant were measured using a Glucose Assay kit (Nanjing Jiancheng Bioengineering Institute). Each experiment was repeated thrice.

Western blotting. 3T3-L1 adipocytes were washed twice with ice-cold PBS and lysed in ice-cold radioimmunoprecipitation assay buffer containing $1 \mathrm{mmol} / \mathrm{l}$ of PMSF and a cocktail of protease inhibitors (1:100; Beyotime Institute of Biotechnology). Next, the total protein concentration of all tissue lysates was determined with a bicinchoninic acid assay protein assay kit (Pierce; Thermo Fisher Scientific, Inc.). Briefly, $20 \mu \mathrm{g}$ protein samples were separated by $10 \%$ SDS-PAGE and transferred to polyvinylidene fluoride membranes (Pall Corporation), and membranes were blocked with PBS containing $10 \%$ non-fat dry milk, overnight at $4^{\circ} \mathrm{C}$. Membranes were then incubated for $12 \mathrm{~h}$ at $4^{\circ} \mathrm{C}$ with antibodies against Nrf2 (1:1,000; cat. no. ab62352; Abcam), HO-1 (1:2,000; cat. no. ab189491; Abcam) and glucose transporter 4 (GLUT-4; 1:2,000; cat. no. ab654; Abcam). Next, membranes were washed and incubated with a goat anti-rabbit IgG horseradish peroxidase-conjugated secondary antibody (1:10,000; cat. no. ab205718; Abcam) for $1 \mathrm{~h}$ at $25^{\circ} \mathrm{C}$. Finally, membranes were visualized using an ECL substrate kit (GE Healthcare Life Sciences) and densitometric analysis was performed using Image Pro-Plus 6.0 software (Media Cybernetics, Inc.). $\beta$-actin served as a reference protein $(1: 5,000$; cat. no. ab8227; Abcam).

Nrf2 small interfering RNA (siRNA) transfection. siRNAs for Nrf2 (si-Nrf2, 5'-AGUUCAAUGAAGCUCAACUTT-3') and negative control [si-negative control (NC), 5'-UUCUCC GAACGUGUCACGUTT-3'] were synthesized by Shanghai GenePharma. IR-3T3-L1 adipocytes were plated at 50\% confluence and transfected with $200 \mathrm{nM}$ si-Nrf2 or si-NC using Lipofectamine ${ }^{\circledR} 3000$ transfection reagent (Invitrogen; Thermo Fisher Scientific, Inc.). At $48 \mathrm{~h}$ after transfection, cells were treated with $100 \mu \mathrm{g} / \mathrm{ml}$ PSP for $48 \mathrm{~h}$.

Statistical analysis. SPSS 19.0 software (IBM, Corps.) was used to perform all statistical analyses. Continuous variables are presented as the mean \pm standard deviation. Significance of the differences among multiple groups was analyzed using 


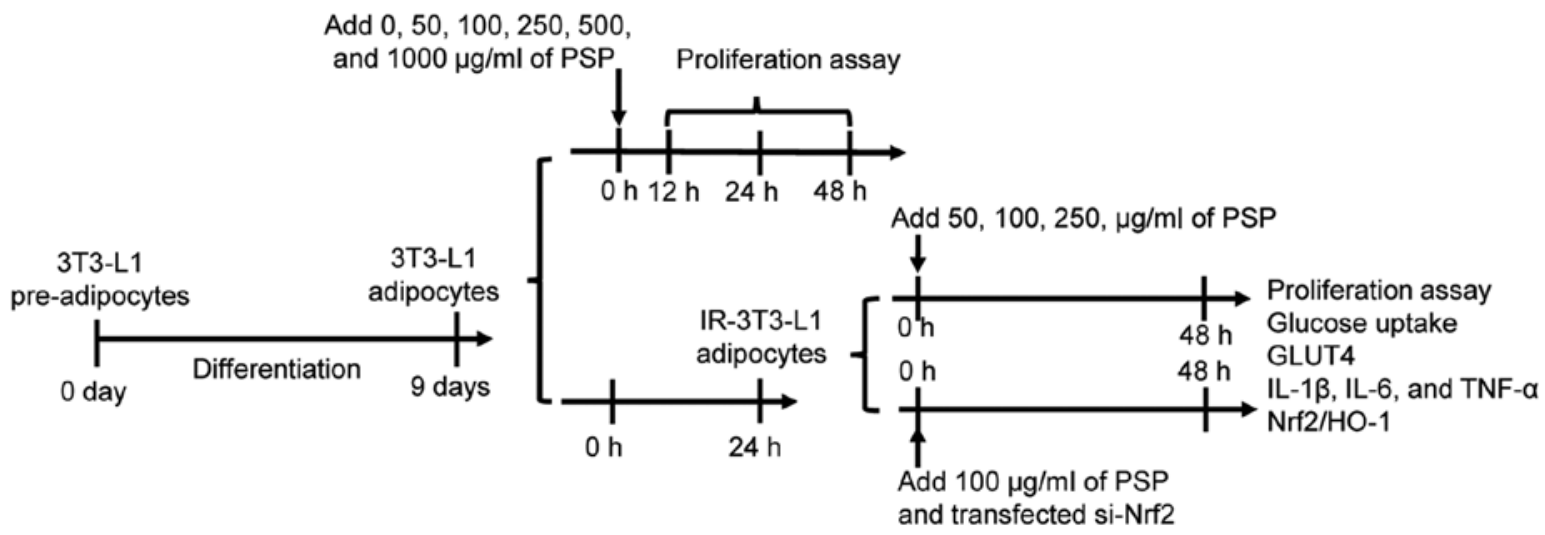

Figure 1. Total experimental schedule. PSP, Polygonatum sibiricum polysaccharide; GLUT4, glucose transporter 4; HO-1, heme-oxygenase-1; IL, interleukin; si, small interfering; Nrf2, nuclear factor erythroid 2-related factor 2; TNF, tumor necrosis factor.
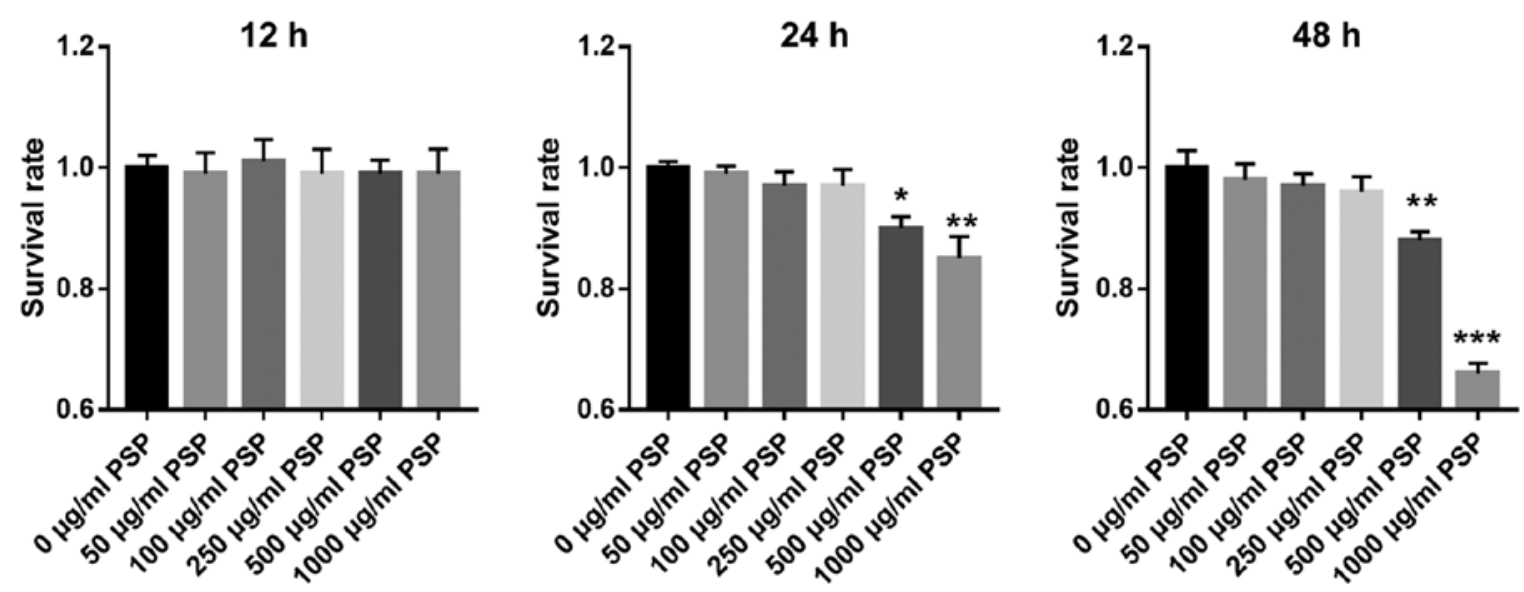

Figure 2. PSP concentration of $<250 \mu \mathrm{g} / \mathrm{ml}$ did not affect the survival of normal-3T3-L1 adipocytes. The survival of normal-3T3-L1 adipocytes was measured at 12,24 and $48 \mathrm{~h}$ via Cell Counting Kit- 8 assay after $0,50,100,250,500$ and $1,000 \mu \mathrm{g} / \mathrm{ml} \mathrm{PSP}$ treatment. ${ }^{*} \mathrm{P}<0.05 ;{ }^{* *} \mathrm{P}<0.01 ;$ and ${ }^{* * * *} \mathrm{P}<0.001 \mathrm{vs} .0 \mu \mathrm{g} / \mathrm{ml}$ group. PSP, Polygonatum sibiricum polysaccharide.

one-way analysis of variance followed by a post-hoc least significant difference test. The significance difference between 2 groups (normal group and IR model group; si-NC group and si-Nrf2 group) was analyzed via the unpaired t-test. $\mathrm{P}<0.05$ were considered to indicate statistically significant.

\section{Results}

Effects of PSP on 3T3-L1 adipocyte proliferation. To determine the effect PSP on the proliferation of normal 3T3-L1 adipocytes, adipocytes were treated with 0, 50, 100, 250, 500 and $1,000 \mu \mathrm{g} / \mathrm{ml}$ PSP. The CCK- 8 assay results showed that 50,100 and $250 \mu \mathrm{g} / \mathrm{ml}$ PSP had no significant effect on the survival of normal 3T3-L1 adipocytes at 12, 24 and $48 \mathrm{~h}$, whereas 500 and $1,000 \mu \mathrm{g} / \mathrm{ml}$ PSP treatments significantly inhibited the survival of normal 3T3-L1 adipocytes in a time-dependent manner from $24 \mathrm{~h}$ onwards $(\mathrm{P}<0.05$; Fig. 2$)$. As per the results, 50, 100 and $250 \mu \mathrm{g} / \mathrm{ml}$ PSP were chosen for subsequent experiments.

PSP treatment alleviates cell survival and glucose uptake in IR-3T3-L1 adipocytes. First, the effect of PSP on proliferation and glucose uptake in IR-3T3-L1 adipocytes was investigated.
CCK-8 assay results showed that IR significantly inhibited the proliferation of 3T3-L1 adipocytes, whereas 50, 100 and $250 \mu \mathrm{g} / \mathrm{ml}$ PSP treatment significantly alleviated the IR effect in IR-3T3-L1 adipocytes $(\mathrm{P}<0.05$; Fig. $3 \mathrm{~A})$. The glucose in the culture medium was significantly increased in the IR model group compared with in the normal cell group and was significantly decreased in the 50,100 and $250 \mu \mathrm{g} / \mathrm{ml}$ PSP treatment group compared with in the IR model group $(\mathrm{P}<0.001$; Fig. 3B). The results suggested that IR significantly inhibited the glucose uptake of 3T3-L1 adipocytes, while 50, 100 and $250 \mu \mathrm{g} / \mathrm{ml}$ PSP treatment significantly alleviated the IR effect in IR-3T3-L1 adipocytes. Moreover, western blot results showed that IR significantly inhibited GLUT-4 expression, whereas 50,100 and $250 \mu \mathrm{g} / \mathrm{ml}$ PSP treatment alleviated this effect in IR-3T3-L1 adipocytes ( $\mathrm{P}<0.001$; Fig. $3 \mathrm{C}$ and 3D). Additionally, proliferation, glucose uptake, GLUT-4 expression exhibited no obvious change between 50, 100 and $250 \mu \mathrm{g} / \mathrm{ml}$ PSP treatment groups.

PSP treatment alleviates inflammatory cytokines in IR-3T3-LI adipocytes. IL-1 $\beta$, IL- 6 and TNF- $\alpha$ levels in the culture supernatant were measured using ELISAs. The results showed that IL-1 $\beta$, IL-6 and TNF- $\alpha$ levels were significantly increased in 

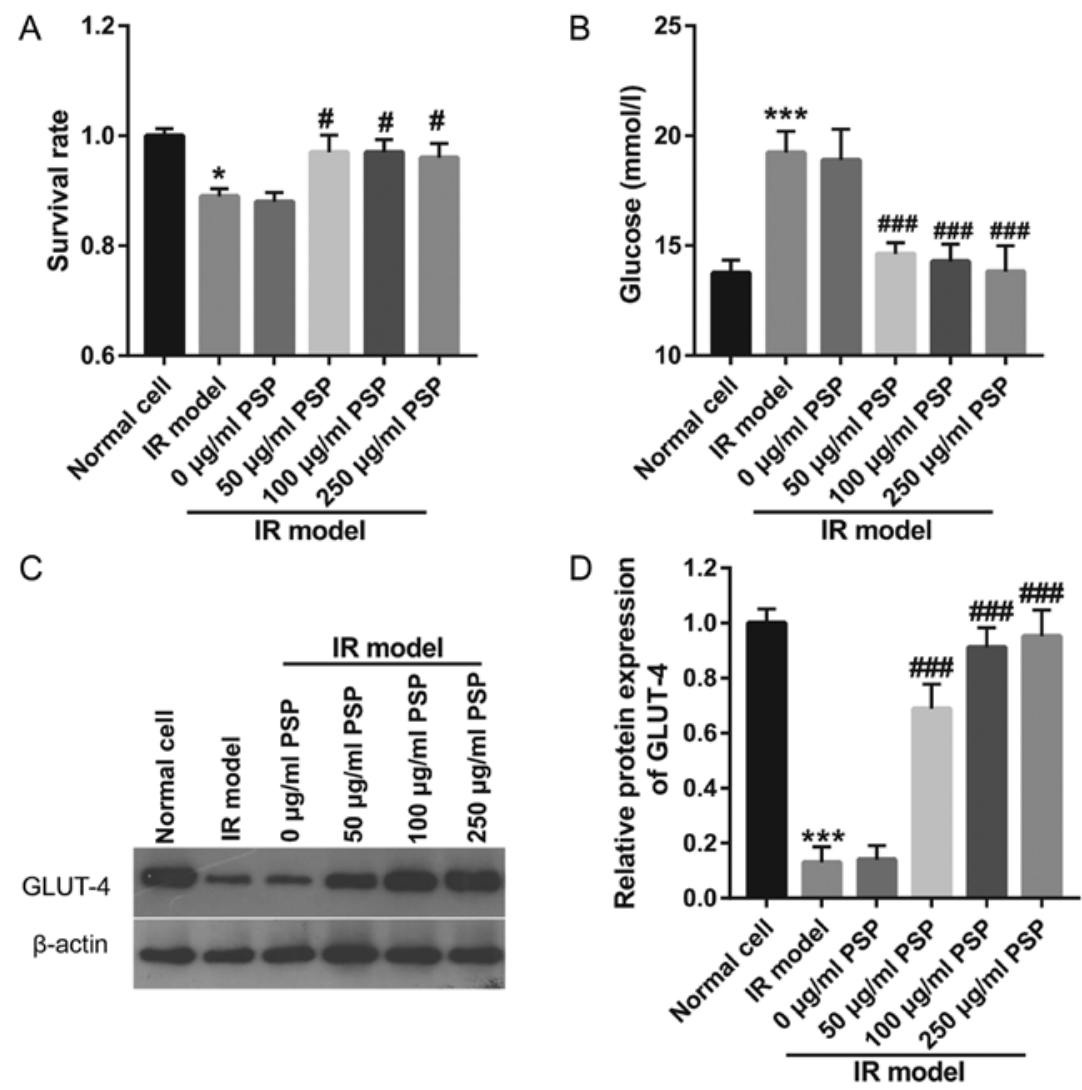

Figure 3. A total of 50,100 and $250 \mu \mathrm{g} / \mathrm{ml}$ PSP treatment improves the proliferation, glucose uptake, and GLUT-4 expression of IR-3T3-L1 adipocytes after treated at $48 \mathrm{~h}$. (A) The proliferation was measured by Cell Counting Kit-8, (B) Glucose concentration in the culture supernatant were measured using a Glucose Assay kit. The smaller the glucose concentration, the better the glucose uptake. (C) GLUT-4 expression in IR-3T3-L1 adipocytes was measured by western blot. (D) The bar graph represents the quantification of respective GLUT-4 protein expression. ${ }^{*} \mathrm{P}<0.05$ and ${ }^{* * *} \mathrm{P}<0.001$ vs. normal cell; ${ }^{\#} \mathrm{P}<0.05$ and

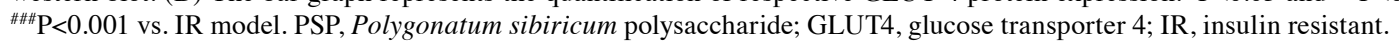
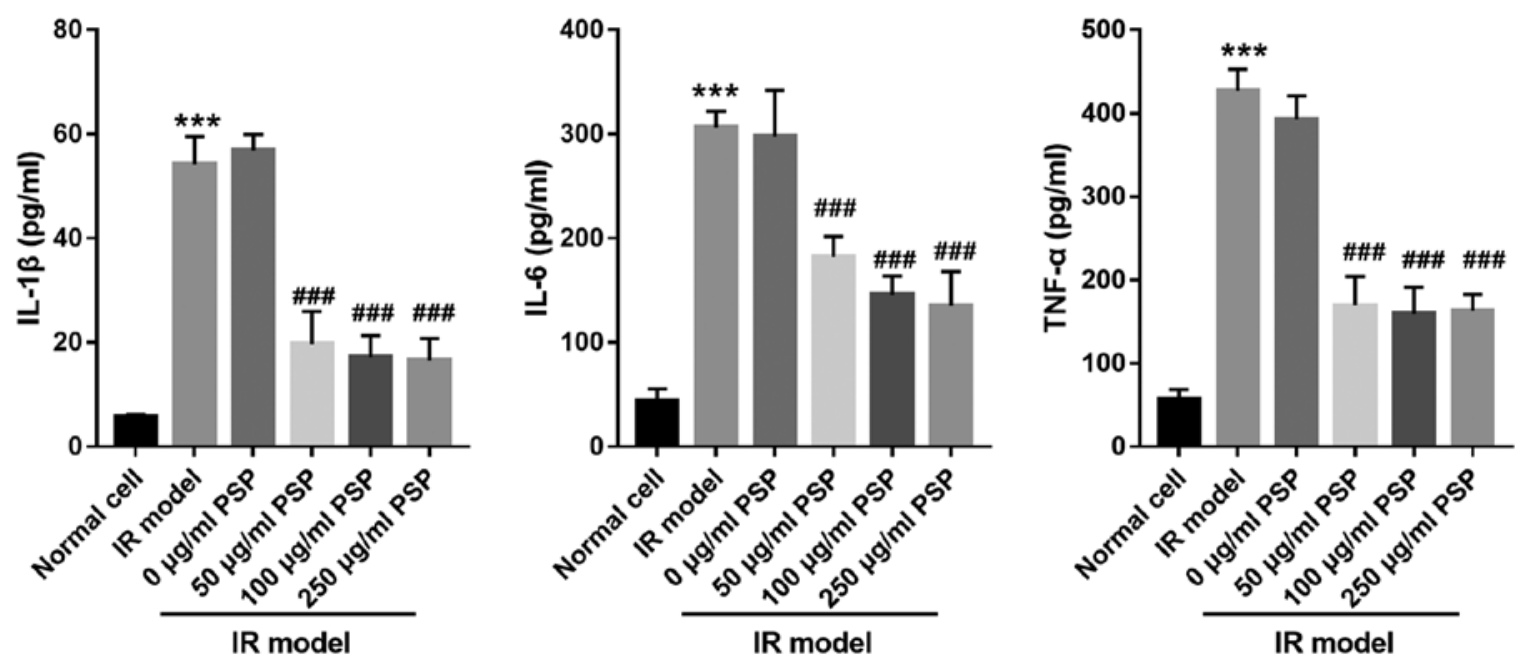

Figure 4. IL-1 $\beta$, IL-6 and TNF- $\alpha$ expression levels are significantly inhibited by PSP treatment in 3T3-L1 adipocyte. IL-1 $\beta$, IL-6, and TNF- $\alpha$ levels in the culture supernatant were measured by ELISA after 50, 100 and $250 \mu \mathrm{g} / \mathrm{ml} \mathrm{PSP}$ treatment at $48 \mathrm{~h}$ in $3 \mathrm{~T} 3-\mathrm{L} 1$ adipocytes. ${ }^{* * *} \mathrm{P}<0.001 \mathrm{vs}$. normal cell; ${ }^{\# \# *} \mathrm{P}<0.001$ vs. IR model. PSP, Polygonatum sibiricum polysaccharide; IL, interleukin; TNF, tumor necrosis factor; IR, insulin resistant.

the IR model group compared with in the normal cell group, and were significantly decreased in the 50,100 and $250 \mu \mathrm{g} / \mathrm{ml} \mathrm{PSP}$ treatment group compared with the IR model group $(\mathrm{P}<0.001$; Fig. 4). Furthermore, IL-1 $\beta$, IL-6 and TNF- $\alpha$ levels exhibited no obvious change between 50, 100 and $250 \mu \mathrm{g} / \mathrm{ml}$ PSP treatment groups.
PSP treatment promotes Nrf2 expression in IR-3T3-LI adipocytes. Next, the underlying mechanism of PSP in IR-3T3-L1 adipocytes was investigated. Western blotting results showed that IR treatment significantly inhibited Nrf2 and HO-1 expression in 3T3-L1 adipocytes ( $\mathrm{P}<0.001$; Fig. 5). However, 50, 100 and $250 \mu \mathrm{g} / \mathrm{ml}$ PSP treatment significantly 
A

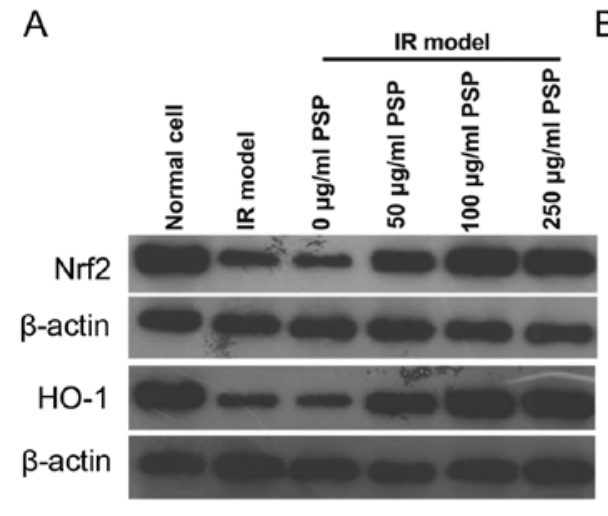

B

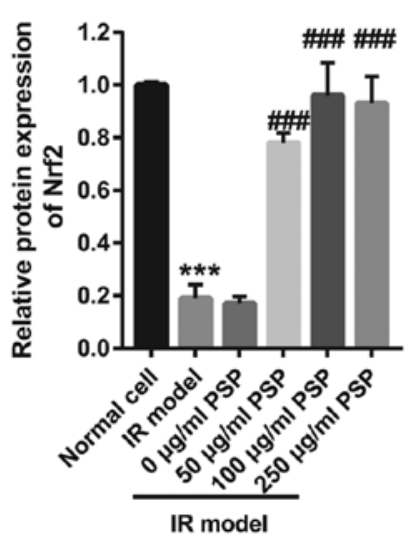

C

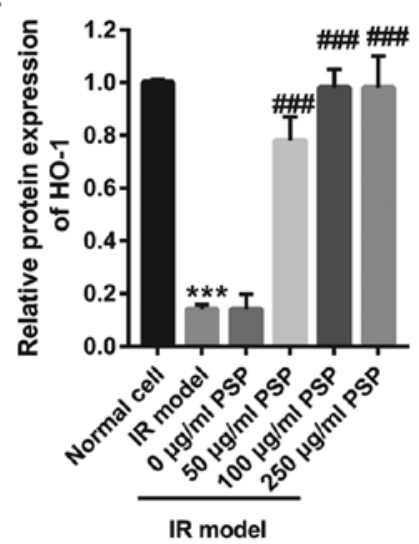

Figure 5. Nrf2 and HO-1 expression, measured by western blot assay, is promoted by 50, 100 and $250 \mu \mathrm{g} / \mathrm{ml}$ PSP treatment in 3T3-L1 adipocytes. (A) Nrf2 and HO-1 expression in IR-3T3-L1 adipocytes were measured by western blot after being treated at $48 \mathrm{~h}$. The bar graphs represent the quantification of respective (B) Nrf2 and (C) HO-1 protein expression. ${ }^{* * *} \mathrm{P}<0.001$ vs. normal cell; ${ }^{\# \#} \mathrm{P}<0.001$ vs. IR model. PSP, Polygonatum sibiricum polysaccharide; HO-1, heme-oxygenase-1; IL, interleukin; Nrf2, nuclear factor erythroid 2-related factor 2; IR, insulin resistant.

A

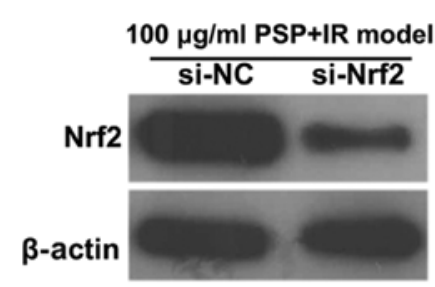

C

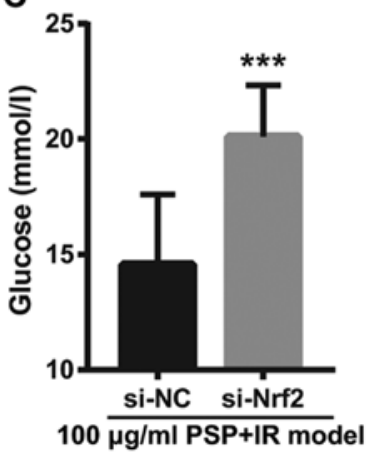

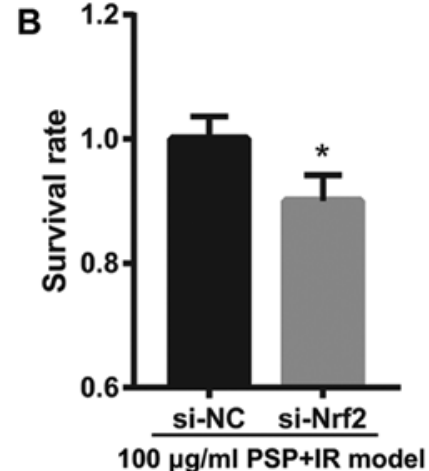

D

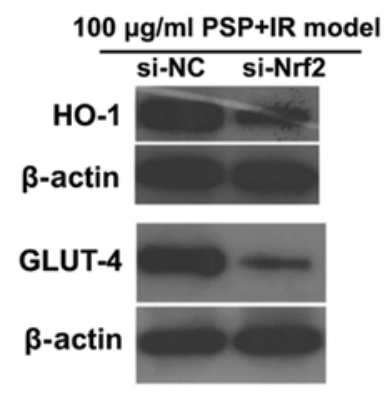

Figure 6. Silencing Nrf2 expression reversed the effects of $100 \mu \mathrm{g} / \mathrm{ml}$ PSP on survival rates and glucose uptake in IR-3T3-L1 adipocytes after treated at $48 \mathrm{~h}$. (A) Nrf2 expression was measured via western blotting after transfecting si-Nrf2 or si-NC into $100 \mu \mathrm{g} / \mathrm{ml}$ PSP-treated IR-3T3-L1 adipocytes. (B) Survival rate, (C) glucose and (D) HO-1 and GLUT-4 expression were measured after transfecting si-Nrf2 or si-NC into $100 \mu \mathrm{g} / \mathrm{ml}$ PSP-treated IR-3T3-L1 adipocytes. ${ }^{*} \mathrm{P}<0.05$ and ${ }^{* * * *} \mathrm{P}<0.001$ vs. siNC. PSP, Polygonatum sibiricum polysaccharide; HO-1, heme-oxygenase-1; si, small interfering; Nrf2, nuclear factor erythroid 2-related factor 2; NC, negative control; IR, insulin resistant.

promoted Nrf2 and HO-1 expression in IR-3T3-L1 adipocytes and reversed the effect of IR on Nrf2 and HO-1 expression (P<0.001; Fig. 5).

Silencing Nrf2 expression reverses the effects of PSP on cell survival and glucose uptake in IR-3T3-L1 adipocytes. To further elucidate the role of $\mathrm{Nrf} 2$ in $100 \mu \mathrm{g} / \mathrm{ml}$ PSP-treated IR-3T3-L1 adipocytes, Nrf2 expression was knocked down using si-Nrf2 in IR-3T3-L1 adipocytes. Nrf2 expression was inhibited by si-Nrf2 when compared with si-NC (Fig. 6A).
Notably, silencing Nrf2 significantly repressed the proliferation of IR-3T3-L1 adipocytes after $100 \mu \mathrm{g} / \mathrm{ml}$ PSP treatment $(\mathrm{P}<0.05$; Fig. 6B). The glucose in the culture medium was also significantly increased in the si-Nrf2 group compared with in the si-NC group $(\mathrm{P}<0.001$; Fig. $6 \mathrm{C})$ and HO- 1 and GLUT-4 expression was inhibited in the si-Nrf2 group when compared with the si-NC group (Fig. 6D). The results suggested that silencing Nrf2 significantly inhibited the glucose uptake of 3T3-L1 adipocytes, thereby reversing the effect of $100 \mu \mathrm{g} / \mathrm{ml}$ PSP. 

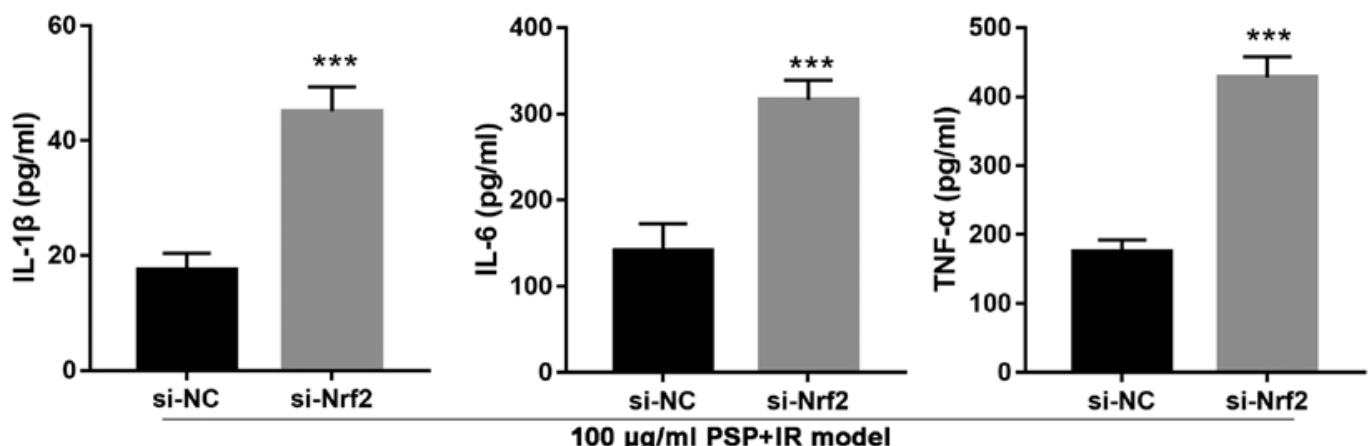

Figure 7. Silencing Nrf2 expression reverses the effects of $100 \mu \mathrm{g} / \mathrm{ml}$ PSP on IL-1 $\beta$, IL- 6 and TNF- $\alpha$ levels. IL-1 $\beta$, IL- 6 , and TNF- $\alpha$ levels in the culture supernatants of IR-3T3-L1 adipocytes were measured by ELISA after treatment at $48 \mathrm{~h}$. ${ }^{* * *} \mathrm{P}<0.001$ vs. the si-NC. PSP, Polygonatum sibiricum polysaccharide; IL, interleukin; si, small interfering; Nrf2, nuclear factor erythroid 2-related factor 2; NC, negative control; TNF, tumor necrosis factor; IR, insulin resistant.

Silencing Nrf2 expression reverses the effects of PSP on inflammatory cytokines in IR-3T3-LI adipocytes IL-1 $\beta$, IL-6 and TNF- $\alpha$ levels in culture supernatants were measured using ELISAs. The results showed that silencing Nrf2 expression significantly promoted IL- $1 \beta$, IL- 6 and TNF- $\alpha$ levels after $100 \mu \mathrm{g} / \mathrm{ml}$ PSP treatment in IR-3T3-L1 adipocytes $(\mathrm{P}<0.001$; Fig. 7). The schematic diagram about PSP-mediated regulation of inflammatory cytokines in high-glucose- and high-insulin-induced 3T3-L1 adipocytes is presented in Fig. 8.

\section{Discussion}

Currently, patients with T2DM are limited in their options for effective therapeutic drugs. Hence, it is important to find novel drugs with respect to T2DM therapy. In this study, it was found that 50, 100 and $250 \mu \mathrm{g} / \mathrm{ml}$ PSP treatments exhibited no significant effects on the survival of normal 3T3-L1 adipocytes; however, the PSP treatments facilitated cell survival, promoted glucose uptake, and inhibited inflammatory expression in IR-3T3-L1 adipocytes. PSP treatment also promoted Nrf2 expression. Reciprocally, silencing Nrf2 expression reduced cell survival, inhibited glucose uptake and promoted inflammatory expression in PSP-treated IR-3T3-L1 adipocytes. Therefore, PSP was verified to play a potential therapeutic effect in T2DM.

The risk of T2DM has been strongly associated with high levels of inflammatory cytokines such as IL-1 $\beta$, IL- 6 , TNF- $\alpha$ and c-reactive protein (23). Dysfunctional adipocytes produce excessive inflammatory cytokines thereby causing IR in skeletal muscle and adipocytes cells and hepatocytes $(22,24)$. IR inhibited glucose uptake by reduced GLUT 4 expression (25). Additionally, obesity-induced IR via the induction of adipocyte inflammation can inhibit glucose uptake and cell proliferation and in adipocytes (26). These above results showed that inflammation can inhibit cell survival and cause IR which can inhibit glucose uptake by reduced GLUT 4 expression. Therefore, an important strategy to treat T2DM is to prevent inflammation. In this study, it was found that IL-1 $\beta$, IL- 6 and TNF- $\alpha$ levels were upregulated, whereas GLUT- 4 expression and glucose uptake were inhibited in IR-3T3-L1 adipocytes, all of which simulate the IR-induced inflammatory microenvironment around adipocytes. It was also found that PSP treatment downregulated IL-1 $\beta$, IL- 6 and TNF- $\alpha$ levels,
Polygonatum sibiricum polysaccharide (PSP) alleviates inflammatory cytokines and promotes glucose uptake in high-glucose- and high-insulin-induced 3T3-L1 adipocytes by promoting Nrf2 expression

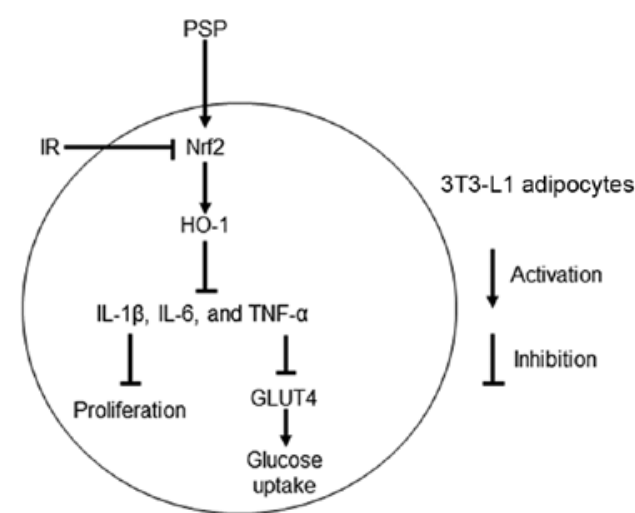

Figure 8. Schematic diagram. GLUT4, glucose transporter 4; HO-1, heme-oxygenase-1; IL, interleukin; si, small interfering; Nrf2, nuclear factor erythroid 2-related factor 2; TNF, tumor necrosis factor; IR, insulin resistance.

facilitated cell survival, and promoted GLUT-4 expression and glucose uptake in IR-3T3-L1 adipocytes. These results suggest that PSP reduces the secretion of inflammatory cytokines which can promote GLUT-4 expression and glucose uptake in IR-3T3-L1 adipocytes and alleviates the IR of 3T3-L1 adipocytes. Similar to the results of the present study that indicated PSP's role in anti-inflammation, previous studies also found that PSP played anti-inflammatory roles via IL-1 $\beta$, IL-6 and TNF- $\alpha(11,14)$. PSP also delayed the progression of diabetic retinopathy and cataracts $(15,16)$, further indicating its therapeutic potential in T2DM.

Nrf2 plays a protective role in T2DM. For example, Actinidia chinensis (Planch.) has been demonstrated to prevent the onset of T2DM by promoting Nrf2 expression, which in-turn improves oxidant and inflammation levels (27). The development of novel and effective Nrf2 activators may provide novel therapeutic drugs for T2DM treatment. The $\mathrm{Nrf} 2 / \mathrm{HO}-1$ signaling pathway is linked to obesity, pancreatic $\beta$ cell damage, IR and glucose metabolism disorders (28). Anthocyanins could ameliorate diabetic-induced retinal cell damage through the Nrf2 / HO-1 signaling pathway (29). In this study, it was found that PSP treatment promoted Nrf2 
and HO-1 expression in IR-3T3-L1 adipocytes. Silencing Nrf2 expression promoted IL-1 $\beta$, IL- 6 and TNF- $\alpha$ secretion and inhibited proliferation, glucose uptake, and HO-1 and GLUT-4 expression, thereby reversing the therapeutic effect of PSP in IR-3T3-L1 adipocytes. These results suggest that PSP alleviates inflammatory cytokines and promotes glucose uptake in IR-3T3-L1 adipocytes by promoting Nrf2 expression.

The present study had two limitations. Firstly, the effects and mechanisms of PSP on T2DM needs to be studied more comprehensively. Secondly, the therapeutic effect of PSP also needs to be clarified in experimental animals and clinical trials.

In conclusion, the present study suggested that PSP has the potential to be used in the treatment of T2DM owing to its inducing effect on Nrf2 expression. However, comprehensive studies are needed that detail the therapeutic effects and mechanisms of PSP in experimental animals and clinical trials in the future

\section{Acknowledgements}

Not applicable.

\section{Funding}

The present study was supported by grants from the youth program of the Natural Science Foundation of Hunan province, China (grant no. 2019JJ5041), the Natural Science Foundation of Hunan University of Chinese Medicine, China (grant no. ZYYDX201740) and the State Administration of Traditional Chinese Medicine of the People's Republic of China Construction Project of Zhang Zhiguo Heritage Studio Related to the Old Chinese Medicine Experts [grant no. (2016)42].

\section{Availability of data and materials}

The datasets used and/or analyzed during the current study are available from the corresponding author on reasonable request.

\section{Authors' contributions}

JC, YZh, XL and GY conceived and designed the present study. JC, YZh, YZu, QT and ZZ developed the methodology. JC, YZh and LY completed the experiment and collected the data. JC, YZh, YZu, QT and ZZ analyzed and interpreted the data. JC and YZh drafted the manuscript. All authors read and approved the final manuscript.

\section{Ethics approval and consent to participate}

Not applicable.

\section{Patient consent for publication}

Not applicable.

\section{Competing interests}

The authors declare that they have no competing interests.

\section{References}

1. NCD Risk Factor Collaboration (NCD-RisC): Worldwide trends in diabetes since 1980: A pooled analysis of 751 population-based studies with 4.4 million participants. Lancet 387: 1513-1530, 2016.

2. He G, Liang Y, Chen Y, Yang W, Liu JS, Yang MQ and Guan R: A hotspots analysis-relation discovery representation model for revealing diabetes mellitus and obesity. BMC Syst Biol 12(Suppl 7): 116, 2018.

3. Scheen AJ and Paquot N: Obesity. A new paradigm for treating obesity and diabetes mellitus. Nat Rev Endocrinol 11: 196-198, 2015.

4. Guilherme A, Virbasius JV, Puri V and Czech MP: Adipocyte dysfunctions linking obesity to insulin resistance and type 2 diabetes. Nat Rev Mol Cell Biol 9: 367-377, 2008.

5. Hasegawa Y, Ikeda K, Chen Y, Alba DL, Stifler D, Shinoda K, Hosono T, Maretich P, Yang Y, Ishigaki Y, et al: Repression of adipose tissue fibrosis through a PRDM16-GTF2IRD1 complex improves systemic glucose homeostasis. Cell Metab 27: 180-194. e6, 2018.

6. Chen L, Lu W and Li Y: Berberine ameliorates type 2 diabetes via modulation of Bifidobacterium species, tumor necrosis factor- $\alpha$, and lipopolysaccharide. Int J Clin Exp Med 9: 9365-9372, 2016.

7. Chen X, Yu J and Shi J: Management of diabetes mellitus with puerarin, a natural isoflavone from pueraria lobata. Am J Chin Med 46: 1771-1789, 2018.

8. Chen M, Liao Z, Lu B, Wang M, Lin L, Zhang S, Li Y, Liu D, Liao Q and Xie Z: Huang-Lian-Jie-Du-Decoction ameliorates hyperglycemia and insulin resistant in association with gut microbiota modulation. Front Microbiol 9: 2380, 2018.

9. Zhao P, Zhao C, Li X, Gao Q, Huang L, Xiao P and Gao W: The genus Polygonatum: A review of ethnopharmacology, phytochemistry and pharmacology. J Ethnopharmacol 214: 274-291, 2018.

10. Cui X, Wang S, Cao H, Guo H, Li Y, Xu F, Zheng M, Xi X and Han C: A review: The bioactivities and pharmacological applications of Polygonatum sibiricum polysaccharides. Molecules 23: E1170, 2018.

11. Liu N, Dong Z, Zhu X, Xu H and Zhao Z: Characterization and protective effect of Polygonatum sibiricum polysaccharide against cyclophosphamide-induced immunosuppression in Balb/c mice. Int J Biol Macromol 107: 796-802, 2018.

12. Yelithao K, Surayot U, Lee JH and You S: RAW264.7 cell activating glucomannans extracted from rhizome of Polygonatum sibiricum. Prev Nutr Food Sci 21: 245-254, 2016.

13. Zeng GF, Zhang ZY, Lu L, Xiao DQ, Xiong CX, Zhao YX and Zong SH: Protective effects of Polygonatum sibiricum polysaccharide on ovariectomy-induced bone loss in rats. J Ethnopharmacol 136: 224-229, 2011.

14. Zhu X, Wu W, Chen X, Yang F, Zhang J and Hou J: Protective effects of Polygonatum sibiricum polysaccharide on acute heart failure in rats 1. Acta Cir Bras 33: 868-878, 2018.

15. Wang Y, Qin S, Pen G, Chen D, Han C, Miao C, Lu B, Su C, Feng S, Li W, et al: Original research: Potential ocular protection and dynamic observation of Polygonatum sibiricum polysaccharide against streptozocin-induced diabetic rats' model. Exp Biol Med (Maywood) 242: 92-101, 2017.

16. Wang Y, Lan C, Liao X, Chen D, Song W and Zhang Q: Polygonatum sibiricum polysaccharide potentially attenuates diabetic retinal injury in a diabetic rat model. J Diabetes Investig 10: 915-924, 2019.

17. Ahmed SM, Luo L, Namani A, Wang XJ and Tang X: Nrf2 signaling pathway: Pivotal roles in inflammation. Biochim Biophys Acta Mol Basis Dis 1863: 585-597, 2017.

18. Lu MC, Ji JA, Jiang ZY and You QD: The Keap1-Nrf2-ARE pathway as a potential preventive and therapeutic target: An Update. Med Res Rev 36: 924-963, 2016.

19. Yoo EJ, Lee HH, Ye BJ, Lee JH, Lee CY, Kang HJ, Jeong GW, Park H, Lim SW, Lee-Kwon W, et al: TonEBP suppresses the HO-1 gene by blocking recruitment of Nrf2 to its promoter. Front Immunol 10: 850, 2019.

20. Cheng Y, Yang C, Luo D, Li X, Le XC and Rong J: N-Propargyl caffeamide skews macrophages towards a resolving M2-like phenotype against myocardial ischemic injury via activating Nrf2/HO-1 pathway and inhibiting NF-kB pathway. Cell Physiol Biochem 47: 2544-2557, 2018.

21. Yu ZW, Li D, Ling WH and Jin TR: Role of nuclear factor (erythroid-derived 2)-like 2 in metabolic homeostasis and insulin action: A novel opportunity for diabetes treatment? World J Diabetes 3: $19-28,2012$ 
22. Liao SP, Wu WQ, Zeng $J$ and $\mathrm{Wu} Y$ : miR-146a regulates inflammatory cytokines and reverses high-glucose- and high-insulin-induced insulin resistance in 3T3-L1 adipocytes by targeting Traf6 through the NF- $\mathrm{\kappa B}$ signaling pathway. Int J Clin Exp Med 11: 1708-1716, 2018.

23. Liu C, Feng X, Li Q, Wang Y, Li Q and Hua M: Adiponectin, TNF- $\alpha$ and inflammatory cytokines and risk of type 2 diabetes: A systematic review and meta-analysis. Cytokine 86: 100-109, 2016.

24. Bastard JP, Maachi M, Lagathu C, Kim MJ, Caron M, Vidal H, Capeau $J$ and Feve B: Recent advances in the relationship between obesity, inflammation, and insulin resistance. Eur Cytokine Netw 17: 4-12, 2006.

25. Su YC, Ou HY, Wu HT, Wu P, Chen YC, Su BH, Shiau AL, Chang CJ and Wu CL: Prothymosin- $\alpha$ overexpression contributes to the development of insulin resistance. J Clin Endocrinol Metab 100: 4114-4123, 2015.
26. Nie Y, Ma RC, Chan JC, Xu H and Xu G: Glucose-dependent insulinotropic peptide impairs insulin signaling via inducing adipocyte inflammation in glucose-dependent insulinotropic peptide receptor-overexpressing adipocytes. FASEB J 26: 2383-2393, 2012

27. Sun L, Li X, Li G, Dai B and Tan W: Actinidia chinensis planch. improves the indices of antioxidant and anti-inflammation status of type 2 diabetes mellitus by activating Keap1 and Nrf2 via the upregulation of microRNA-424. Oxid Med Cell Longev 2017: 7038789, 2017.

28. Uruno A, Yagishita Y and Yamamoto M: The Keap1-Nrf2 system and diabetes mellitus. Arch Biochem Biophys 566: 76-84, 2015.

29. Song Y, Huang L and Yu J: Effects of blueberry anthocyanins on retinal oxidative stress and inflammation in diabetes through Nrf2/HO-1 signaling. J Neuroimmunol 301: 1-6, 2016. 